\title{
Cutaneous Drug Reactions in a Tertiary Government Hospital in the Philippines 2009-2011
}

\author{
Carmela Augusta F. Dayrit, Clarita C. Maaño and Michael Lawrenz F. Co \\ Section of Dermatology, Department of Medicine, College of Medicine and Philippine General Hospital, University of the Philippines Manila
}

\begin{abstract}
Objectives. To determine the frequency of Cutaneous Drug Reactions (CDRs), their associated drugs, and morphological presentations in the Philippine General Hospital (PGH) from 2009 to 2011 and to identify new or uncommon drugs causing CDRs.

Methods. This is a 3-year retrospective record review. The Naranjo algorithm was used to score drug causality.

Results. One hundred and forty-three (143) patient records were retrieved, with 218 associated drugs identified. The most common drug classes were antibiotics (29\%), anti-tuberculosis medications (17\%), and NSAIDs (9\%). The most common drugs were isoniazid, rifampicin, pyrazinamide, ethambutol (HRZE) combination drug (9\%); amoxicillin (6\%); and cotrimoxazole (5\%). A morbiliform reaction (49\%) was the most common morphological presentation. There were several identified drugs, including anti-fungals and beta-blockers, which caused a single drug reaction but had a sufficient Naranjo score to warrant inclusion.
\end{abstract}

Conclusions. The most common drug classes, drugs, and cutaneous morphological presentations found in this study are similar to those seen in existing literature. However, there were several drugs identified causing single drug reactions. This may reflect the need for improved documentation, diagnosis, and follow-up of CDR cases in the PGH.

Key Words: drug reaction, adverse drug reaction, Naranjo Score, Philippines

\section{Introduction}

An Adverse Drug Reaction (ADR) can be defined as a harmful or seriously unpleasant effect of a drug at therapeutic doses. ADRs include all unintended pharmacologic effects of a drug, excluding abuse of the

Paper presented at the Internal Medicine Research Forum, November 2012, PGH ER Complex. Poster presented at the Philippine General Hospital Research Forum, November 2013, PGH Atrium.

Corresponding author: Carmela Augusta F. Dayrit, MD

Section of Dermatology

Department of Medicine

Philippine General Hospital

University of the Philippines Manila

Taft Avenue, Ermita, Manila 1000 Philippines

Telefax No: +632 6311422

Email: miya_day@yahoo.com drug, or errors in administration. ${ }^{1,2}$ A suspected drug can be identified to cause the ADR by the timing, pattern of illness, and re-challenge tests. ${ }^{1}$ It is important to know the ADRs to specific drugs for patient safety, drug development, and responsible prescribing practices.

Among all the in-patient referrals to the Section of Dermatology in the Philippine General Hospital (PGH) from 1984 to 1987 , drug reactions ranked $2^{\text {nd }}(15.5 \%)$ as the most common reasons for referral. When grouped by age, drug reactions ranked $1^{\text {st }}(20.2 \%)$ in the adult age group, $4^{\text {th }}$ $(10.5 \%)$ in the adolescent age group, and $5^{\text {th }}(7.5 \%)$ in the geriatric age group. ${ }^{3}$

According to the ADR Outline of the PGH for 1998, $86 \%$ of all ADRs seen at the PGH Out Patient Department (OPD), were found to have cutaneous manifestations. Cutaneous Drug Eruptions (CDRs) include morphologies like morbiliform or maculopapular lesions; urticaria and angioedema; pigmentation or fixed drug eruptions (FDE); vesicular, bullous, pustular, and lichenoid reactions; and erythroderma.

With some slight differences in frequency, the most common implicated drugs causing CDRs include antibiotics (penicillin, sulfonamides), anti-convulsants (phenytoin, phenobarbital, carbamazepine), analgesics (paracetamol, aspirin, naproxen, mefenamic acid) and anti-tuberculosis drugs (rifampicin, pyrazinamide, isoniazid). ${ }^{5-9}$

This retrospective study aims to determine the frequency of Cutaneous Drug Reactions (CDRs), their associated drugs, and morphological presentations as seen in the PGH from the year 2009 to 2011 and to identify any new or uncommon drugs causing CDRs.

\section{Methods}

Study design

This is a descriptive, retrospective record review conducted at the University of the Philippines-Philippine General Hospital (UP-PGH), a tertiary government hospital based in Metro Manila.

Inclusion Criteria

Patients, of any age and sex, seen or referred to Dermatology and/or Allergy for a new onset of CDR from January 2009 to December 2011 and scored with the Naranjo 
algorithm to have a definite, probable, or possible drug reaction.

\section{Exclusion Criteria}

Patients with missing charts or records, those with a doubtful drug reaction as scored with the Naranjo algorithm, those who were initially assessed to have an ADR or CDR but were proven otherwise were excluded, and those assessed to have a hypersensitivity reaction without a history of drug intake and cases of intentional and accidental poisoning, drug overdose, or drug abuse, were excluded.

\section{Data collection}

The outpatient and ward referral census logbooks of the UP-PGH Section of Dermatology and Section of Allergy from January 2009 to December 2011 were scanned for all patients with assessments of drug reactions including: ADR, CDR, adverse drug event (ADE), fixed drug eruption (FDE), drug hypersensitivity, erythroderma secondary to drug, photoallergic reaction secondary to drug, Erythema Multiforme (EM), Stevens-Johnson Syndrome (SJS), Toxic Epidermal Necrolysis (TEN), and other diagnoses with a drug being the suspected cause of a drug reaction. Patient case record numbers were used to retrieve their charts from the Medical Records Section. A co-investigator reviewed all available charts and used the Narajo algorithm to determine drug causality. Only definite, probable, and possible ADRs were included for further data extraction. The primary investigator then reviewed the charts, used the Naranjo Algorithm to re-score the cases, and extracted the data using data extraction forms from the charts that were still scored definite, probable, and possible ADRs.

\section{The Naranjo Algorithm}

The Naranjo Algorithm consists of a set of questions on previous reports of reactions to the suspected drug, timing of the reaction, re-challenge tests, possible alternative causes, drug tests, dose alteration effects, previous reactions to similar drugs, and the presence of objective evidence. ${ }^{10}$ These questions have a corresponding point system and a score of 9 or more defines a definite ADR; a score of 5-8 a probable ADR; a score of 1-4 a possible ADR; and a score of 0 a doubtful ADR. Similar algorithms for adverse drug reactions have been described however, consensual, content, and concurrent validity has been established for the Naranjo Algorithm and hence was utilized in this study (Appendix).

\section{Statistical analysis}

Information from the data extraction sheet was encoded in Microsoft Excel spreadsheet. Stata software (version 12) was used to analyze the data.

Descriptive statistics were used to summarize the data. Categorical/nominal data was presented in terms of frequency and percentage/proportion. Fisher's exact test was used to study the relationship between variables.
Ethics

This study was submitted to the University of the Philippines, Manila Research Ethics Board (UPMREB) for ethical review and approval. All the data retrieved from the logbooks and charts was kept confidential. Unique numerical codes were assigned to each patient and no personal identifiers such as names or initials was used. Only the researchers have access to the study database.

\section{Results}

There were 311 ADR cases identified from the OPD and ward referral logbooks of the PGH Section of Dermatology and Allergy from January 2009 to December 2011. Of the identified cases, 191 (61.4\%) charts were retrieved and $48 \mathrm{did}$ not fit the inclusion criteria. This left a total of 143 records for further analysis.

Majority of the patients with CDRs were adults with a mean age of 37.4 years old $(S D=18.82)$, were female, and were seen as out-patients. Majority had a reaction to a single drug (Table 1). Most of the patients were seen by Dermatology (38\%), followed by Allergy (20\%). Other specialties who first saw the patients with CDRs include: General Medicine, Pediatrics, Medicine Subspecialites (IDS, Endocrinology, Hematology, Oncology, Pulmonology), Family Medicine, Neurology, Ophthalmology, Obstetrics and Gynecology, Otorhinolaryngology, Orthopedics, and Dentistry.

Table 1. Patient Demographics ( $n=143)$.

\begin{tabular}{lcc}
\hline \multicolumn{1}{c}{ Age Group } & Frequency & $\%$ \\
\hline \multicolumn{1}{c}{ Age } & 22 & \\
Pediatric (<18 years) & 112 & $15 \%$ \\
Adult (19-64 years) & 9 & $78 \%$ \\
Geriatric (>65 years) & & $6 \%$ \\
Sex & 58 & \\
Male $\quad 85$ & $41 \%$ \\
Female Patient status & 112 & $59 \%$ \\
Out-patient & 31 & $78 \%$ \\
In-patient Number of drugs & & $22 \%$ \\
1 drug & 95 & $66 \%$ \\
2 drugs & 29 & $20 \%$ \\
3 or more drugs & 19 & $14 \%$ \\
\hline
\end{tabular}

The mean Naranjo score was 3.25 with a standard deviation of 1.62. This score translated to a "possible drug reaction."

Of the 143 cases identified, a total of 218 associated drugs were found. The top 5 drug classes were antibiotics, anti-TB medications, NSAIDs, anti-convulsants, and antihypertensives. (Table 2).The HRZE combination (9\%) was the most common drug identified. Amoxicillin (6\%), cotrimoxazole $(5 \%)$, cloxacillin $(4 \%)$, and carbamazepine $(4 \%)$ were the other more common drugs associated with CDRs. 
Table 2. Drugs associated with Cutaneous Drug Reactions, OPD and ward referrals, PGH Section of Dermatology, 20092011.

\begin{tabular}{|c|c|c|}
\hline & Frequency & $\%$ \\
\hline Antibiotics & 63 & $29 \%$ \\
\hline Amoxicillin & (13) & \\
\hline Co-trimoxazole & (11) & \\
\hline Cloxacillin & (9) & \\
\hline Co-amoxiclav & (4) & \\
\hline Clindamycin & (4) & \\
\hline Cefalexin & (3) & \\
\hline Erythromycin & (3) & \\
\hline Azithromycin & (2) & \\
\hline Ceftazidime & (2) & \\
\hline Levofloxacin & (2) & \\
\hline Penicillin & (2) & \\
\hline Others & $(8)$ & \\
\hline Anti-TB & 38 & $17 \%$ \\
\hline HRZE & $(20)$ & \\
\hline HRZ & (3) & \\
\hline Isoniazid & (3) & \\
\hline Rifampicin & (3) & \\
\hline Streptomycin & (3) & \\
\hline HRE & (2) & \\
\hline Pyrazinamide & (2) & \\
\hline $\mathrm{RE}$ & (1) & \\
\hline Ethambutol & (1) & \\
\hline NSAIDs & 19 & $9 \%$ \\
\hline Mefenamic Acid & (6) & \\
\hline Aspirin & (3) & \\
\hline Ibuprofen & (3) & \\
\hline Diclofenac & (2) & \\
\hline Ibuprofen+Paracetamol & (2) & \\
\hline Naproxen & (2) & \\
\hline Meloxicam & (1) & \\
\hline Anti-convulsant & 18 & $8 \%$ \\
\hline Carbamazepine & (8) & \\
\hline Phenobarbital & (5) & \\
\hline Phenytoin & (4) & \\
\hline Oxcarbazepine & (1) & \\
\hline Anti-HPN & 10 & $5 \%$ \\
\hline Amlodipine & (2) & \\
\hline Losartan & (2) & \\
\hline Others & (6) & \\
\hline Chemotherapy & 7 & $4 \%$ \\
\hline Imatinib & $(2)$ & \\
\hline Others & (5) & \\
\hline Steriod & 6 & $3 \%$ \\
\hline Prednisone & (5) & \\
\hline Dexamethasone & (1) & \\
\hline OTC & 5 & $2 \%$ \\
\hline Carbocisteine & $(2)$ & \\
\hline Phenylephrine & $(2)$ & \\
\hline Nafarin & $(1)$ & \\
\hline Herbal & 5 & $2 \%$ \\
\hline Xanthine oxidase inhibitor: Allopurinol & 4 & $2 \%$ \\
\hline Anti-platelet & 3 & $1 \%$ \\
\hline Anagrelide & (2) & \\
\hline Dipyridamole & (1) & \\
\hline Paracetamol & 3 & $1 \%$ \\
\hline Statins & 3 & $1 \%$ \\
\hline Simvastatin & (2) & \\
\hline Atorvastatin & (1) & \\
\hline Vitamins & 3 & $1 \%$ \\
\hline Vitamin B complex & $(2)$ & \\
\hline Ascorbic Acid & (1) & \\
\hline Bigunanide: Metformin & 2 & $1 \%$ \\
\hline COX-2 Inhibitor: Celecoxib & 2 & $1 \%$ \\
\hline H2 blocker: Ranitidine & 2 & $1 \%$ \\
\hline PPI: Omeprazole & 2 & $1 \%$ \\
\hline Others & 23 & $11 \%$ \\
\hline Total & 218 & $100 \%$ \\
\hline
\end{tabular}

Majority of the CDRs were morbiliform or maculopapular. Other common morphological presentations included urticaria-angioedema, bullous reactions, pigmentation, and erythroderma (Figure 1).

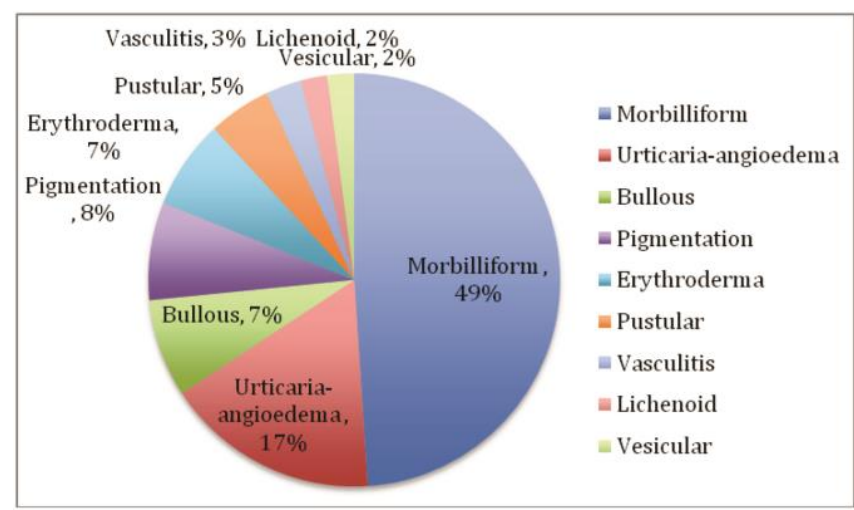

Figure 1. Morphological presentations of Cutaneous Drug Reactions ( $\mathrm{n}=143)$

Fisher's exact test was done to check for associations between the drug classes and the specific morphological presentations. The top 5 morphological presentations with the top 5 drug classes identified were analyzed. There was no association between morbiliform drug reactions and erythroderma with any of the top 5 drug classes. NSAIDs were more likely to be associated with urticaria-angioedema $(p$-value $=0.002)($ Table 3$) . \quad$ Anti-convulsants were more likely to be associated with bullous drug reactions ( $\mathrm{p}$-value= 0.001) (Table 4). Antibiotics were more likely to be associated with pigmentation $(p$-value $=0.050)($ Table 5$)$.

Table 3. Association between Top 5 Drug Classes and Urticaria-Angioedema (Fisher's exact test)

\begin{tabular}{lcccc}
\hline \multirow{1}{*}{ Drugs } & \multicolumn{2}{c}{ Urticaria $C D R$} & \multirow{2}{*}{ Total } & \\
\cline { 2 - 3 } & $\mathrm{n}=24$ & $\mathrm{~N}=119$ & $\mathrm{~N}=143$ & \\
& $9(37.5)$ & $41(34.4)$ & $50(35.0)$ & 0.817 \\
\hline Antibiotic & $7(29.2)$ & $7(5.9)$ & $14(9.8)$ & 0.002 \\
NSAIDs & $6(25.0)$ & $25(21.0)$ & $31(21.7)$ & 0.786 \\
Anti-TB & $1(4.2)$ & $17(14.3)$ & $18(12.6)$ & 0.309 \\
Anticonvulsant & $2(8.3)$ & $10(8.4)$ & $12(8.4)$ & 1.000 \\
Antihypertension & & & & \\
\hline
\end{tabular}

Table 4. Association between Top 5 Drug Classes and Bullous Drug Reaction (Fisher's exact test)

\begin{tabular}{lcccc}
\hline \multirow{1}{*}{\multicolumn{1}{c}{ Drugs }} & \multicolumn{2}{c}{ Bullous CDR } & \multirow{2}{*}{ Total } & p-value \\
\cline { 2 - 3 } & $\mathrm{n}=11$ & $\mathrm{n}=132$ & $\mathrm{~N}=143$ & \\
\hline Antibiotic & $3(27.3)$ & $47(35.6)$ & $50(35.0)$ & 0.747 \\
NSAIDs & $0(0.0)$ & $14(10.6)$ & $14(9.8)$ & 0.601 \\
Anti-TB & $3(27.3)$ & $28(21.2)$ & $31(21.7)$ & 0.704 \\
Anticonvulsant & $6(54.6)$ & $12(9.1)$ & $18(12.6)$ & 0.001 \\
Antihypertension & $0(0.0)$ & $12(9.1)$ & $12(8.4)$ & 0.599 \\
\hline
\end{tabular}


Table 5. Association between Top 5 Drug Classes and Pigmentation

\begin{tabular}{lcccc}
\hline \multirow{2}{*}{\multicolumn{1}{c}{ Drugs }} & \multicolumn{2}{c}{ Pigmentation $C D R$} & \multirow{2}{*}{ Total } & p-value \\
\cline { 2 - 3 } & $\mathrm{n}=11$ & $\mathrm{n}=132$ & $\mathrm{~N}=143$ & \\
\hline Antibiotic & $7(63.6)$ & $43(32.6)$ & $50(35.0)$ & 0.050 \\
NSAIDs & $1(9.1)$ & $13(9.8)$ & $14(9.8)$ & 1.000 \\
Anti-TB & $1(9.1)$ & $30(22.7)$ & $31(21.7)$ & 0.457 \\
Anticonvulsant & $0(0.0)$ & $18(13.6)$ & $18(12.6)$ & 0.359 \\
Antihypertension & $1(9.1)$ & $11(8.3)$ & $12(8.4)$ & 1.000 \\
\hline
\end{tabular}

Time of Appearance and Morphological Presentation

For morbiliform eruptions, the mean time of appearance was 24.53 days $(S D=93.66)$. However, there were two outliers with times of appearance of 120 and 730 days. These two cases were not excluded since they received a Naranjo score of 3 and 4, respectively. Excluding these two values, mean time of appearance was reduced to 10.95 days.

Urticaria-angioedema had the fastest time of appearance compared to the other morphological presentations with a mean of 7.9 days $(S D=14.81)$. Time of appearance of bullous drug reactions had a mean of 13.1 days $(\mathrm{SD}=9.52)$.

Pigmentation had the longest time of appearance with a mean of 62.71 days $(\mathrm{SD}=82.75)$. There were two outliers, both with a time of appearance of 180 days. These cases were also not excluded since both got a Naranjo score of 4, with skin punch biopsy findings consistent with a drug reaction. Without the two outliers, the mean was 15.8 days. Time of appearance of erythroderma had a mean of 25.8 days $(\mathrm{SD}=$ 38.2).

Most CDRs had a time of appearance between 1-7 days (33\%), 8-15 days (16\%), and 16-30 days (12\%) (Table 6).

Table 6. Time between Drug Intake and Cutaneous Drug Reaction

\begin{tabular}{lcc}
\hline & Frequency & $\%$ \\
\hline Immediate/few minutes & 16 & $7 \%$ \\
$<24$ hours & 10 & $5 \%$ \\
$1-7$ days & 72 & $33 \%$ \\
8-15 days & 35 & $16 \%$ \\
16-30 days & 27 & $12 \%$ \\
$31-60$ days & 10 & $5 \%$ \\
$>60$ days & 9 & $4 \%$ \\
No data & 39 & $18 \%$ \\
TOTAL & 218 & $100 \%$ \\
\hline
\end{tabular}

Relation of CDRs to Comorbid Conditions

Age group, gender, concurrent diseases, and family history of atopy were related to the appearance of each morphological type of drug reaction (morbiliform, urticaria, bullous, pigmentation, and erythroderma).

The Fisher's Exact t-test was used to check for correlation between the morphologic presentations of CDRs and the presence of co-morbid conditions including atopy, renal disease, autoimmune disease, diabetes, and hypertension. There were no significant associations found.

\section{Discussion}

A total of 218 associated drugs were found to be associated with adverse drug reactions. The most common drug classes were antibiotics (29\%), anti-TB medications $(17 \%)$, NSAIDs $(9 \%)$, and anti-convulsants $(8 \%)$; and the most common drugs were HRZE combination (9\%), amoxicillin (6\%), and co-trimoxazole $(5 \%)$, cloxacillin $(4 \%)$, and carbamazepine $(4 \%)$. These findings were similar to previous studies where the most common implicated drugs were antibiotics (penicillin, sulfonamides), anti-convulsants (phenytoin, phenobarbital, carbamazepine), and antituberculosis drugs (rifampicin, pyrazinamide, isoniazid). ${ }^{5-9}$

Morbiliform reactions comprised $49 \%$ of the CDRs. Other morphological types included urticaria-angioedema (17\%), bullous drug reactions (7.5\%), pigmentation $(7.5 \%)$, and erythroderma (7\%). Again, these findings were similar to previous studies, which found that the morbilliform type was the most common clinical presentation of CDRs. Other of the more frequently encountered types of CDRs included fixed drug reaction (also pigmentation), SJS/TEN (also bullous reactions), and urticaria-angioedema.,11-13

Urticaria-angioedema was found to be significantly associated with NSAIDs ( $p=0.002)$; bullous reactions with anti-convulsants $\quad(p=0.001)$; and pigmentation with antibiotics $(p=0.050)$. This reflects similar reports that NSAIDs, together with antibiotics, are the drugs most commonly associated with urticaria; ${ }^{14,15}$ antibiotics, together with NSAIDs, are the most commonly associated with pigmentation and fixed drug eruptions; ${ }^{12}$ and anticonvulsants (carbamazepine, phenytoin, and phenobarbital), together with sulfonamide antibiotics, NSAIDs, and allopurinol, are most commonly associated with SJS/TEN. ${ }^{11,16}$ This may have implications when presented with patients with a specific morphologic type of drug reaction, i.e., the doctor should investigate further about history of intake of these particular drug classes.

Most CDRs had a time of appearance between 1-7 days (33\%). Urticaria-angioedema had the shortest mean time of appearance of 7.9 days $(S D=14.81)$ while pigmentation had the longest mean time of appearance of 62.71 days $(\mathrm{SD}=$ 82.75). Urticaria-angioedema has been found to a type I immediate hypersensitivity reaction and this reflects the fastest time between drug intake and appearance of the drug reaction as seen in this study.

Previous studies identified patient factors such as female sex, adults, and the presence of concurrent diseases and infections such as liver or renal disease, HIV infection, asthma, and systemic lupus erythematosus to be related to ADRs. ${ }^{5,17}$ In this study, there were no significant associations found between each morphological type of drug reaction and possible patient risk factors such as age, gender, comorbid conditions, or family medical history. There was no significant relationship found between CDR morphologic type and concurrent illnesses like bronchial asthma and 
atopy, hypertension and diabetes, renal disease, and autoimmune disease.

Finally, it was found that the mean Naranjo score in included drugs was 3.25 with a standard deviation of 1.62 . This score translated to a "possible drug reaction" only. This finding suggests that improvement in the documentation and management of cases assessed to have drug reactions may be needed. Author recommendations include the use of available patient adverse drug reaction protocols to ensure detailed history taking, physical examination, and proper documentation of patients; and the adequate utilization of diagnostic tests such as drug re-challenge tests and skin punch biopsies.

\section{Conclusion}

This study showed that the drugs and drug classes associated with drug reactions and the typical morphological presentations of CDRs are consistent with those in existing literature. It also confirmed the association between specific drugs and particular morphologic presentations, such as NSAIDs with urticaria, antibiotics with pigmentation, and anti-convulsants with bullous drug reactions. However, possible related risk factors on developing drug reactions were not identified.

Using the Naranjo algorithm, it was found that the mean Naranjo score was low corresponding to a "possible drug reaction" only. This may reflect the way drug reactions are documented and imply that diagnosis, management, and follow-up of patients with CDRs should be improved.

\section{References}

1. Edwards AR, Aronson JK. Adverse drug reactions: definitions, diagnosis, and management. Lancet. 2000; 356(9237):1255-9.

2. Joint Task Force on Practice Parameters; American Academy of Allergy, Asthma and Immunology; American College of Allergy, Asthma and Immunology; Joint Council of Allergy, Asthma and Immunology. Solensky R, Khan D. Drug allergy: an updated practice parameter. Ann Allergy Asthma Immunol. 2010; 105(4):259-73.

3. Tianco EAV, Gutierrez GT. A survey of the adult and pediatric ward referrals to the University of the Philippines-Philippine General Hospital Dermatology Section from January 1984 to December 1987. Phillip J Intern Med. 1989; 27:119-27.

4. Adverse Drug Reactions Outline 1998. Section of Allergy and Immunology. UP-PGH.

5. Sharma VK, Sethuraman GG. Adverse cutaneous reactions to drugs: an overview. J Postgrad Med [Online]. 1996 [cited 2013 Jan]. Available from http://www.jpgmonline.com/text.asp?1996/42/1/15/464.

6. Saka B, Kombate K, Mouhari-Toure A, Akakpo S, Tchangai-Walla K, Pitche P. Stevens-Johnson syndrome and toxic epidermal necrolysis in a teaching hospital in Lome, Togo: a retrospective study of 89 cases. Med Trop. 2010; 70(3):255-8

7. Guillano GJ, Visitacion LR. A review of cutaneous drug hypersensitivity reactions in a tertiary referral center: a four-year retrospective study from January 2000 to December 2003, Davao Medical Center, Davao City, Philippines. J Phillip Dermatol Soc. 2006; 15: 44-50.

8. Bigby M, Jick S, Jick H, Arndt K. Drug-induced cutaneous reactions. A report from the Boston Collaborative Drug Surveillance Program on 15,438 consecutive inpatients, 1975 to 1982. JAMA. 1986; 256(24):3358-63.
9. Danza A, Lopez M, Vola M, Alvarez-Rocha A. Adverse cutaneous reactions to drugs among hospitalized patients: a one-year surveillance. Rev Med Chil. 2010; 138(11):1403-9.

10. Naranjo CA, Busto U, Sellers EM, et al. A method for estimating the probability of adverse drug reactions. Clin Pharmacol Ther. 1981; 30(2):239-45.

11. Gosiengfiao V, Alberto N. Cutaneous drug reactions and their causative agents seen at the Philippine General Hospital Section of Dermatology from January 2001 to March 2003. J Phillip Dermatol Soc. 2005; 14(1):4952.

12. Raksha MP, Marfatia YS. Clinical study of cutaneous drug eruptions in 200 patients. Indian J Dermatol Venereol Leprol. 2008; 74(1):80.

13. Lee HY, Tay LK, Thirumoorthy T, Pang SM. Cutaneous adverse drug reactions in hospitalized patients. Singapore Med J. 2010; 51(10):767-74.

14. Roujeau JC. Clinical aspects of skin reactions to NSAIDs. Scand J Rheumatol Suppl. 1987; 65:131-4.

15. Tan EK, Grattan CE. Drug-induced urticaria. Expert Opin Drug Saf. 2004; 3(5):471-84.

16. Hebert AA, Ralston JP. Cutaneous reactions to anticonvulsant medications. J Clin Psychiatry. 2001; 62 Suppl 14:2226.

17. Nayak S, Achariva B. Adverse cutaneous drug reaction. Indian J Dermatol. 2008; 53(1):2-8. 


\section{Appendix}

\section{NARANJO ALGORITHM}

1. Are there previous conclusive reports on this reaction? Yes (+1) No (0) Do not know or not done (0)

2. Did the adverse event appear after the suspected drug was given? Yes (+2) No (-1) Do not know or not done (0)

3. Did the adverse reaction improve when the drug was discontinued or a specific antagonist was given? Yes (+1) No (0) Do not know or not done (0)

4. Did the adverse reaction appear when the drug was readministered? Yes (+2) No (-2) Do not know or not done (0)

5. Are there alternative causes that could have caused the reaction? Yes (-1) No (+2) Do not know or not done (0)

6. Did the reaction reappear when a placebo was given? Yes (-1) No (+1) Do not know or not done (0)

7. Was the drug detected in any body fluid in toxic concentrations? Yes (+1) No (0) Do not know or not done (0)

8. Was the reaction more severe when the dose was increased, or less severe when the dose was decreased? Yes (+1) No (0) Do not know or not done (0)

9. Did the patient have a similar reaction to the same or similar drugs in any previous exposure? Yes (+1) No (0) Do not know or not done (0)

10. Was the adverse event confirmed by any objective evidence? Yes (+1) No (0) Do not know or not done (0)

\section{Scoring}

$>9=$ definite ADR

$5-8=$ probable ADR

1-4 = possible ADR

$0=$ doubtful ADR

Naranjo CA, Busto U, Sellers EM, Sandor P, Ruiz I, Roberts EA, Janecek E, Domecq C, and Greenblatt DJ. A method for estimating the probability of adverse drug reactions. Clinical Pharmacology and Therapeutics. 1981;30:239-245. 